\title{
Indoor Localization with Multi-objective Selection of Radiomap Models
}

\author{
Rafael Alexandrou ${ }^{(\bowtie)}$, Harris Papadopoulos ${ }^{(\bowtie)}$, \\ and Andreas Konstantinidis $\left.{ }^{(}\right)$ \\ Frederick University, 7 Y. Frederickou Street Pallouriotisa, 1036 Nicosia, Cyprus \\ \{res.ar,com.ph, com.ca\}@frederick.ac.cy
}

\begin{abstract}
Over the last years, Indoor Localization Systems (ILS) evolved, due to the inability of Global Positioning Systems (GPS) to localize in indoor environments. A variety of studies tackle indoor localization with technologies such as Bluetooth Beacons and RFID that require costly installation, or techniques such as Google Wi-Fi/Cell DB and fingerprinting that leverage from the already existing Wi-FI and telecommunication infrastructure. Additionally, recent studies attempt to solve the same problem using Bio-Inspired techniques, such as Artificial Neural Networks (ANNs) and Deep Neural Networks (DNN). In this paper, we introduce a Multi-Objective Optimization Radiomap Modelling (MOO-RM) based ILS. The MOO-RM ILS divides the dataset into clusters using a K-Means algorithm and trains ANN models on the data of each cluster. The resulting models are fed into a MultiObjective Evolutionary Algorithm based on Decomposition (MOEA/D), which minimizes the required storage space and the localization error, simultaneously. Our experimental studies demonstrate the superiority of the proposed approach on real datasets of Wi-Fi traces with respect to various existing techniques.
\end{abstract}

Keywords: Indoor Localization $\cdot$ Smartphones $\cdot$ Fingerprinting $\cdot$ Artificial Neural Networks · Multi-Objective Optimization

\section{Introduction}

Currently, Global Navigation Satellite Systems (GNSS), such as GPS, are unable to localize accurately in indoor environments due to the satellite signal attenuation while passing through solid objects. This has led to an increased interest for alternative localization techniques by the scientific community. In particular, the indoor localization community has proposed a variety of solutions that include technologies such as Bluetooth Beacons (BLE), Infrared, Li-Fi technologies, RFID, Sensor Networks and their combinations, for localizing a device in an indoor environment with a fine-grain accuracy [1]. These techniques, however, require the deployment of specialized equipment such as antennas, beacons, and custom transmitters a priori [2], which is time consuming and costly. 
In order to alleviate the aforementioned challenges, a variety of Indoor Localization Systems (ILS) that rely on geolocation data retrieved from the existing infrastructure of a building, such as wireless signals, have been implemented. These ILS, such as Google, Indoo.rs, Navizon, IndoorAtlas, ByteLight and Anyplace $^{1}[3]$ have managed to provide accurate indoor localization of a user without the need of any additional hardware.

Geolocation database entries act as reference points for the localization tasks. A comparison between those reference points and a sensed point from a smartphone device, either on the service (Server-Side) or the smartphone itself (ClientSide), can help determine a user's location within an area. As [4] suggests, ServerSide violates user's privacy, since calculations, that are processed on the server, reveal the actual location of a user to the service. Contrary, such concern does not apply for Client-Side, since the service only knows the area that surrounds the user and not the user's actual location. However, since the main processing of the ILS is done on the smartphone, this leads to performance concerns, mainly, due to the vast amount of data being downloaded and processed locally.

In this paper, we examine the possibility of providing the user with a variety of solutions based on his preferences, by introducing a novel Multi-Objective Optimization Radiomap Modelling (MOO-RM) based ILS. The solutions include Artificial Neural Network (ANN) models, associated to clusters of location data, which are generated using K-Means and can be used to localize users. Then, a Multi-Objective Evolutionary Algorithm Based on Decomposition (MOEA/D) is used to minimize the storage space requirements on the smartphone device and the localization error, by selecting a near-optimal set of models from the set of all models that represent the whole location-based dataset.

The rest of this paper is organized as follows. Section 2 covers the background of indoor localization and provides solutions in the literature that are similar to our proposed method. Section 4 presents the proposed ILS method, which is evaluated in Sect.5. Finally, Sect. 6 discusses future work.

\section{Related Work}

In this section, background on indoor localization that lies at the foundations of the proposed approach is introduced.

\subsection{Indoor Localization with Smartphones}

A wide range of technologies dealing with localization in outdoor and indoor environments is provided in the literature. For outdoor environments, Global Navigation Systems (GNSS), such as GPS and Galileo, are considered the leading technologies for localization. However, they require high energy consumption and their signal attenuation while passing through solid objects, such as concrete walls, is negatively affecting navigation in indoor environments. A variety of indoor localization solutions [1] have been proposed including technologies such

\footnotetext{
${ }^{1}$ Available at: http://anyplace.cs.ucy.ac.cy/.
} 
as BLE sensors, visual or acoustic analysis, RFID, Wireless Sensor Networks, laser and LiFi, IMUs and their combinations into hybrid systems. Even though the performance of the aforementioned suggest positive votes, most of these techniques require the costly deployment of additional equipment a priori to localization. Contrary, the indoor localization community introduced localization approaches, such as Cell/Wi-Fi Database and Wi-Fi Fingerprinting, that rely on existing Wi-Fi infrastructure already deployed in most buildings.

Wi-Fi Fingerprints construct a database of radio signals from Wi-Fi Access Points (Wi-Fi APs) within the area. In the literature, Anyplace [5-7] achieved the second highest known accuracy [8], with an average error of 1.96 meters. In particular, Anyplace is divided into two phases: The offline phase, or "logging" phase, records the Wi-Fi Fingerprints, which are Received Signal Strength indications (RSSi) of Wi-Fi APs at certain locations $(x, y)$ of a building, into an $N \times M$ matrix known as a radiomap. The radiomap itself consists of $N$ unique fingerprints and $M \mathrm{Wi}-\mathrm{Fi}$ APs. In the online phase, or "localization" phase, a smartphone user observes the RSSi from the surrounding Wi-Fi APs and compares it against the radiomap using either K-Nearest Neighbors (KNN) or Weighted K-Nearest Neighbors (WKNN) to find the best match. Finally, Anyplace provides two different approaches: In Server-Side Fingerprinting (SS), the main localization process happens on a server that has unlimited energy, storage and processing budget. Therefore, the smartphone user requires little network messaging and minimal energy consumption to localize. However, this approach violates the user's privacy, since the server knows the actual location of the user. Contrary, Client-Side Fingerprinting (CS) focuses on processing locally on the smartphone to eliminate the privacy concerns. However, this leads to high resource consumption on the client device.

In our previous work [9], we proposed a CS fingerprinting approach that utilizes Artificial Neural Networks (ANNs) to train a model representing the whole radiomap data. Even though this approach reduces the energy consumption and storage space required on the smartphone, while preserving user's privacy and maintaining an acceptable localization error compared to conventional fingerprinting techniques, we realized that storage space requirements are still high for smartphone devices. In this work, we investigate the trade-off between storage space requirements and localization error by providing multiple solutions, that consist of a subset of ANN models corresponding to clusters within the radiomap. A variety of solutions will allow users to localize in indoor environments based on their preferences (e.g. preserve battery/storage levels on the device, or minimize localization error during localization).

\subsection{Smartphone Indoor Localization Applications}

In [10], authors present a deep learning-based fingerprinting localization schema. Similarly,[11] presents a deep-learning approach that utilizes deep architectures and Channel State Information (CSI) on fingerprinting for localization. Additionally, authors of [12] present the WiDeep approach that uses a probabilistic denoising auto-encoder. All three aforementioned research studies provide solutions for improving the fingerprinting technique. However, the use of deep neural 
networks requires high volume of resources during localization, due to networks' structure complexity.

Furthermore, the indoor localization problem is, also, tackled as an optimization problem, in the literature. An indoor localization problem is formulated in [13] and optimized with a Particle Swarm Optimization (PSO) approach, named JADE. Likewise, [14] proposes a fingerprinting localization and tracking system with PSO and Kalman Filter (KF). Finally, the authors of [15] present MILos, a multi-objective indoor localization service that utilizes a Multi-Objective Evolutionary Algorithm Based on Decomposition (MOEA/D) to maximize the coverage and minimize the energy consumption at the same time. The latter optimizes coverage and energy consumption objectives on conventional fingerprinting techniques, whereas our proposed method optimizes the storage space required on the device and the localization error using ANN models, which are clustered using K-Means.

\section{System Overview}

\subsection{System Model}

We assume an area $A$ divided into several building areas $A_{1}, . ., A_{n}$, which consist of several floors $F_{1}, . ., F_{m}$ (see Fig. 1). Each $A_{i}$ and its corresponding $F_{j}$ contain a finite set of $(x, y)$ points and is covered by a set of Wi-Fi Access Points $\left\{a p_{1}, a p_{2}, \cdots, a p_{M}\right\}$, each covering $a$ planar points. Area $A_{i}$ is not necessarily continuous and can be considered as the joint area of all $a p_{i} \in A P$ (i.e., global coverage). Each $a p_{i}$ has a unique ID (i.e., MAC address) that is publicly broadcasted and passively received by anyone moving in the $a$ points of $a p_{i}$. The signal intensity at which the ID of $a p_{i}$ is received at location $(x, y)$, is termed the Received Signal Strength $(R S S)$ of $a p_{i}$ at $(x, y)$, having a value in the range $[-30 \ldots-110] d B$.

Let a static (cloud-based) localization service $s$ hosting an $N \times M$ table, coined RadioMap $(R M)$, which records the RSS of the $a p_{i} \in A P$ broadcasts at specified $(x, y) \in A$ locations. When an $a p_{i}$ is not seen at a certain $(x, y)$ the $R M$ records " -110 " in its respective cell. A user $u$ localizes through the indoor positioning service $s$, using the ID and RSS broadcasts of surrounding $a p_{i} \in A P$ while moving. This information is termed, hereafter, RSS Vector or Fingerprint $\left(V_{u}\right)$ of user $u$, which changes from location to location and over time. Contrary to $R M$ rows having $M$ attributes, $V_{u}$ has only $M^{\prime} \ll M$ attributes.

\subsection{Research Goal and Metrics}

Research Goal. Allow a smartphone user to localize with fine accuracy and minimum storage resources by selecting a subset of ANN models representing clusters of Radiomap data.

The efficiency of the proposed technique to achieve the above research goal is measured by the Localization Error, and the Storage Space Requirements on the client device $u$. 


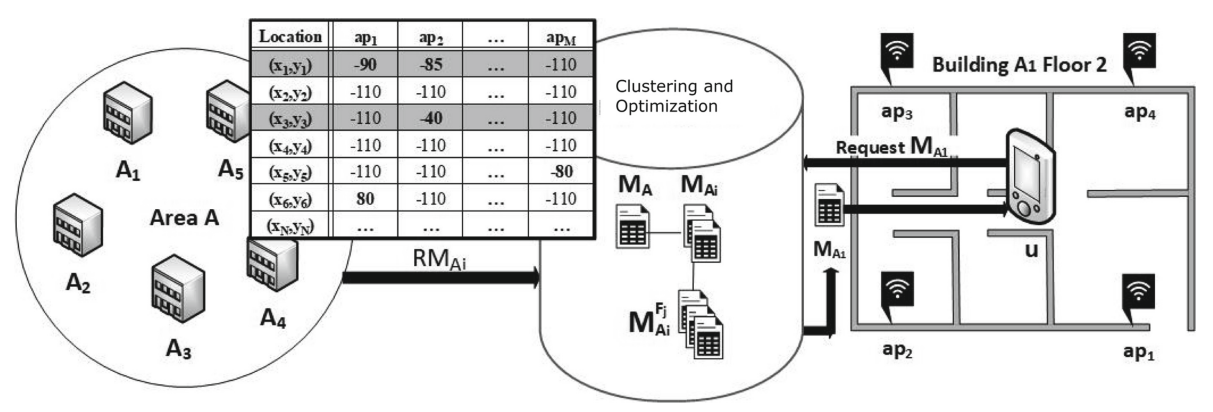

Fig. 1. System model

The Localization Error is defined as the Haversine distance (in $m$ ), between the predicted location $\left(x_{2}, y_{2}\right)$ and the actual location $\left(x_{1}, y_{1}\right)$ of $u$,

$$
d=2 r \times \arcsin \left(\sqrt{\sin ^{2}\left(\frac{x_{2}-x_{1}}{2}\right)+\cos \left(x_{1}\right) \cos \left(x_{2}\right) \sin ^{2}\left(\frac{y_{2}-y_{1}}{2}\right)}\right) / 1000
$$

where $r=6371 \mathrm{~km}$ is the radius of earth.

Storage Space $\left(\right.$ Size $\left._{s}\right)$ is the storage space required on the client device in order to perform indoor localization.

\section{Proposed Methodology}

Our proposed Indoor Localization Method is divided into four phases, namely, Data Clustering, Modeling, Optimization, and Decision Making. Each phase is explained next.

\subsection{Data Clustering}

Data Clustering utilizes a K-Means algorithm to divide the dataset into clusters. In particular, K-Means is a vector quantization method that is widely used in data mining and aims to partition $n$ observations into $k$ clusters based on their distances.

In our proposed method, K-Means is applied to the multi-dimensional fingerprinting dataset for forming clusters with respect to the whole vector, considering both the sensed Received Signal Strength Intensity (RSSI) and the mapped geo-location. Figure 2 provides an example of the resulting clusters for the three floors of our dataset.

\subsection{Modeling}

After the clustering process, each cluster is modelled with an Artificial Neural Network (ANN). In particular, the trained models are able to predict the user's 


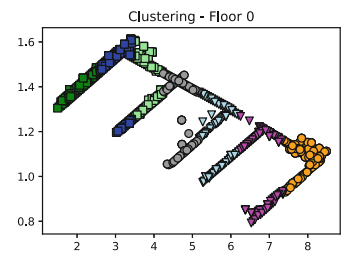

(a) Floor o Clusters

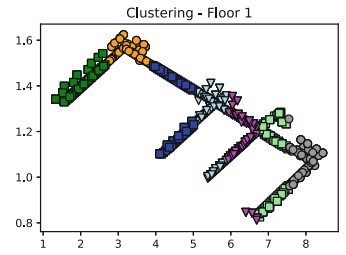

(b) Floor 1 Clusters

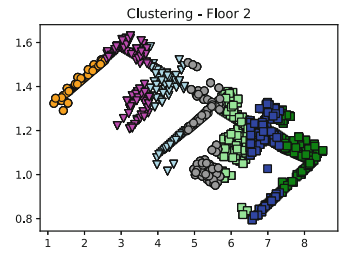

(c) Floor 2 Clusters

Fig. 2. Geographical representation of clusters

current location based on a vector $V_{u}$ that contains the RSSIs of the user's surrounding Wi-Fi APs, arranged in the same order for all patterns. The models used were fully connected feed-forward ANNs with sigmoid hidden and linear output activation functions and were trained with a stochastic gradient descent optimizer called Adam. Implementation was performed using the Scikit-Learn library in a Python environment with the ANN regression algorithm MLPRegressor. Following a trial and error approach for the network's structure led to the average optimum scenario of 100 hidden units on a single hidden layer.

The trained models were extracted and stored on the server in order to be readily available for being sent to a smartphone device. The model extraction was performed manually since Scikit-learn uses a python tool to extract models commonly known as pickle (or its updated version called joblib) which extracts unnecessary information along with the model. This lead to a larger model file compared to the original $R M s$, which conflicts with one of the major goals of the proposed method i.e. to minimize the storage space required on the smartphone device. Therefore, the model's coefficients and intercepts were extracted and saved into two small files. On the smartphone device, both files were loaded and used to reconstruct the model locally, before localization. Note that this reconstruction does not imply re-training the model, since the coefficients and intercepts are known from the training phase.

\subsection{Multi-Objective Optimization}

A Multi-objective Optimization Problem (MOP) can be mathematically formulated as

$$
\text { minimize } F(X)=\left(f_{1}(X), \ldots, f_{k}(X)\right) \text {, subject to } X \in \Omega
$$

where $\Omega$ is the decision space and $X \in \Omega$ is a decision vector. $F(X)$ consists of $k$ objective functions $f_{i}: \Omega \rightarrow R, i=1, \ldots, k$, where $R^{k}$ is the objective space. The objectives often conflict with each other and improving on one objective may lead to deterioration of another. Thus, no single solution exists that can optimize all objectives simultaneously. In that case, the best trade-off solutions, called the set of Pareto optimal (or non-dominated) solutions, is often required by a decision maker. The image of the Pareto Set (PS) in the objective space is called the Pareto Front (PF). 
In this paper the MOP aims at minimizing the storage space required on the smartphone device and the localization error, and it is tacked by the MultiObjective Evolutionary Algorithm Based on Decomposition (MOEA/D). The initial population is generated by a random generator that provides binary vectors, of length equal to number of clusters, where 1 and 0 denote that the corresponding model is included to the set or not. Finally, MOEA/D follows a single point crossover and the termination condition is set to $n$ maximum generations.

\subsection{Decision Making}

Decision Making is the last phase and has access to a set of solutions instead of a single one. It relies on the user's input and the smartphone's available storage. Considering a device that has limited storage availability, the user is asked to decide whether he/she wants to save storage by sacrificing localization accuracy. The user's choice will dictate the need for a more accurate or less storage-dependent model subset. Finally, note that the less storage-dependent model subset implies reduced accuracy within the whole area but not within the part of the area that is covered by the subset. (e.g. model subsets that include only 1 model will be able to predict user's location accurately in $14 \%$ of the whole area).

\section{Experimental Evaluation}

\section{$5.1 \quad$ Dataset}

For our experimental evaluation, we used a real dataset consisting of $\approx 45,000$ reference fingerprints taken from $\approx 120 \mathrm{Wi}$-Fi APs installed in three floors of a building in Cyprus. Therefore, it contains three radiomaps that correspond to the three floors of the building. Firstly, each floor's radiomap was divided into smaller clusters, which further divided into a training and a test set corresponding to $80 \%$ and $20 \%$ of the data, respectively. The training sets were used to model each cluster's data and the models were evaluated in the corresponding test sets.

The proposed indoor localization method is evaluated in terms of Localization Error and Storage Space required on the device, as defined in Sect.3.2.

\subsection{Evaluation Metrics}

The metrics utilized during the optimization phase are defined as follows:

Hypervolume $\left(I_{H}\right)$ indicates the area dominated by at least one solution in an obtained non-dominated set $A$. Therefore, high $I_{H}$ suggests better diversity. The metric is formally defined as

$$
I_{H}(A)=\int_{x \in U_{x \in A}} \cdots \int_{H V\left(f(x), f^{*}\right)} 1 . d z
$$


where $H V(f(x), f *)=\left[f_{1}(x), f_{1}^{*}\right] \times \ldots \times\left[f_{m}(x), f_{m}^{*}\right]$ is the Cartesian product of the closed intervals $\left[f_{i}(x), f_{i}^{*}\right], i=1, . ., m$. Since we consider minimization objectives the reference point $f^{*}=\left(f_{1}^{*} \ldots f_{m}^{*}\right)$ is the ideal worst point.

Number of Non-dominated Solutions $(N D S)$ is the number of nondominated solutions in set $A$.

$$
N D S(A)=|A|
$$

C-Metric is defined by the percentage of non-dominated solutions of set $A$ with respect to solutions of a non-dominated set $B$ and can be annotated as $C(A, B)$.

\subsection{Experimental Study 1: Number of Clusters}

In the first experimental study, we evaluated the K-means algorithm for various cluster sizes in terms of localization error. Note that various cluster sizes were examined but only three are presented, which are representative for all cases. Mainly, the focus of this experimental study is to provide information regarding the expected output of the whole set of models.

Figure 3 shows the comparison of three cluster sizes, in terms of localization error and storage space requirements. Figure 3a shows a similar average localization error of clusters for the three different clusters sizes at each floor. Figure $3 \mathrm{~b}$ examines the storage space requirements on the smartphone device and shows that for floor 0 and floor 2 are similar in all three cases. Contrary, floor 1 results show that the lowest storage space required is achieved using seven clusters. In general, seven clusters seem to provide promising results, for both objectives, and therefore it is adopted in all experimental studies that follow.

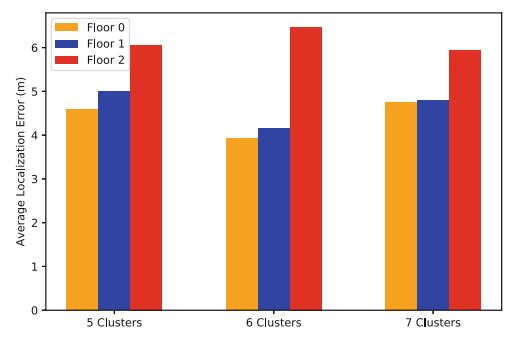

(a) Average Localization Error

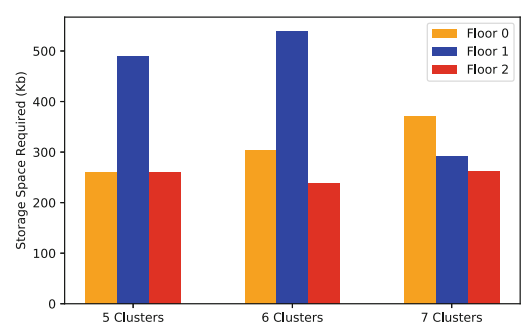

(b) Storage Space

Fig. 3. Clusters performance

\subsection{Experimental Study 2: Optimization Integrity}

The second experimental study focuses on ensuring the integrity of MOEA/D while minimizing the two conflicting objectives with respect to the state-of-theart in MOO, Non-Dominated Sorting Genetic Algorithm II (NSGA-II). Table 1 
shows a comparison between NSGA-II and MOEA/D in terms of the metrics defined in Sect.5.2. The results show similar performance with respect to $I_{H}$ and $C$, but it seems that MOEA/D provides a higher number of $N D S$ to the decision maker.

Table 1. NSGA-II(A) vs MOEA/D(B)

\begin{tabular}{l|l|l|l|l|l|l}
\hline Floor & $I_{H}(A)$ & $N D S(A)$ & $C(A, B)$ & $I_{H}(B)$ & $N D S(B)$ & $C(B, A)$ \\
\hline 0 & 0.846 & 7 & 0.571 & 0.819 & 9 & 0.666 \\
\hline 1 & 0.778 & 14 & 0.714 & 0.834 & 22 & 0.545 \\
\hline 2 & 0.73 & 14 & 0.8 & 0.73 & 20 & 0.8 \\
\hline
\end{tabular}

\subsection{Experimental Study 3: Localization Error and Storage Space}

In order to ensure the usability of the proposed indoor localization method, we evaluate our models with the floor level test set obtained in Sect. 4.2 and over three randomly generated paths within the area, namely, a visitor, a professor, and a student path. Finally, our proposed method was evaluated over an error-dependent scenario (solution with minimum localization error), a storagedependent scenario (solution with minimum storage space required), a conventional fingerprinting technique such as Weighted K-Nearest Neighbors (WKNN), and an Artificial Neural Network (ANN) model generated from the whole training set (without clustering).

Figure 4 provides the Pareto Front (PF) solutions obtained from the optimization phase. Additionally, the solutions of WKNN and ANN single model are provided within the same figure for each floor, as baselines for minimum localization error and minimum storage requirements, respectively. As shown in Fig. 4a, for floor 0, all MOO-RM PF solutions require less storage space than WKNN, and $71 \%$ of the MOO-RM PF solutions require less storage space than the ANN model, as well. As expected, since the WKNN solution is one of our baselines, it has the minimum localization error, whereas the majority of MOORM PF solutions are similar to the localization error of the ANN. Figure $4 \mathrm{~b}$ shows that all MOO-RM PF solutions require less storage than WKNN and $64 \%$ of MOO-RM PF need less storage space than the ANN, for floor 1. Additionally, various MOO-RM PF solutions are close to the localization error provided by the ANN. Lastly, Fig. 4c represents the MOO-RM PF solutions for floor 2. The results demonstrate the superiority of the proposed approach in real life large scale datasets. In particular, all MOO-RM PF solutions require less storage space compared to the WKNN and ANN baselines. Additionally, there are solutions that are similar to WKNN's localization error with a significant decrease in storage space requirements.

Figure 5 shows the results on the user defined paths using the two extreme solutions in the PF obtained by MOO-RM that is: i) Error-Dependent solution 


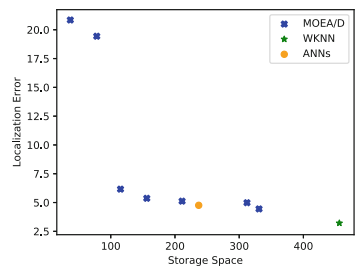

(a) Floor 0

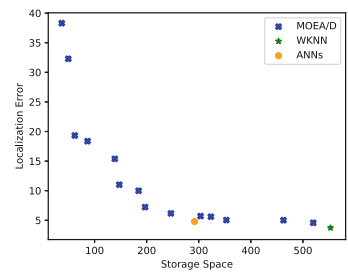

(b) Floor 1

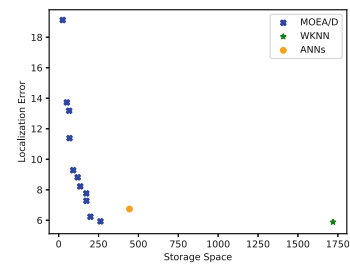

(c) Floor 2

Fig. 4. Floor level evaluation

provides the least possible localization error without any storage space concerns, ii) Storage-Dependent solution provides the least possible storage space on the smartphone device without considering the localization error levels. The results demonstrate that in almost all cases, MOO-RM maintains an acceptable localization error and less storage space requirements, compared to WKNN and ANN when considering the Error-Dependent solution. Additionally, the StorageDependent solution provides a localization error, which is higher compared to WKNN and ANN, offering however a storage space decrease of $99 \%$.

\subsection{Experimental Study 4: Building Level}

In this experimental study, the proposed MOO-RM was evaluated over a building level dataset. In particular, we utilized all 21 models of the three floors, and we examined the performance of MOO-RM on a building level (larger dataset) than on a floor level.

The comparison between MOO-RM, the conventional fingerprinting (WKNN), and the ANN model is shown in Table 2. In particular, the proposed method has an increase of $6 \%$ in terms of localization error and minimizes the storage space by $56 \%$, compared to WKNN. Additionally, MOO-RM decreases the localization error by $54 \%$ and the storage space requirements by $67 \%$, compared to the ANN single model. The ANN model attempts to predict the radiomap of the whole building area $A$, while MOO-RM utilizes a MOO selection between the 21 clusters converging to better localization error.

Table 2. Building level comparison

\begin{tabular}{l|l|l|l}
\hline & WKNN & ANNs & MOO-RM \\
\hline Error $(\mathrm{m})$ & 4.274 & 9.96 & 4.55 \\
\hline Storage $(\mathrm{Kb})$ & 2729 & 3697 & 1203 \\
\hline
\end{tabular}



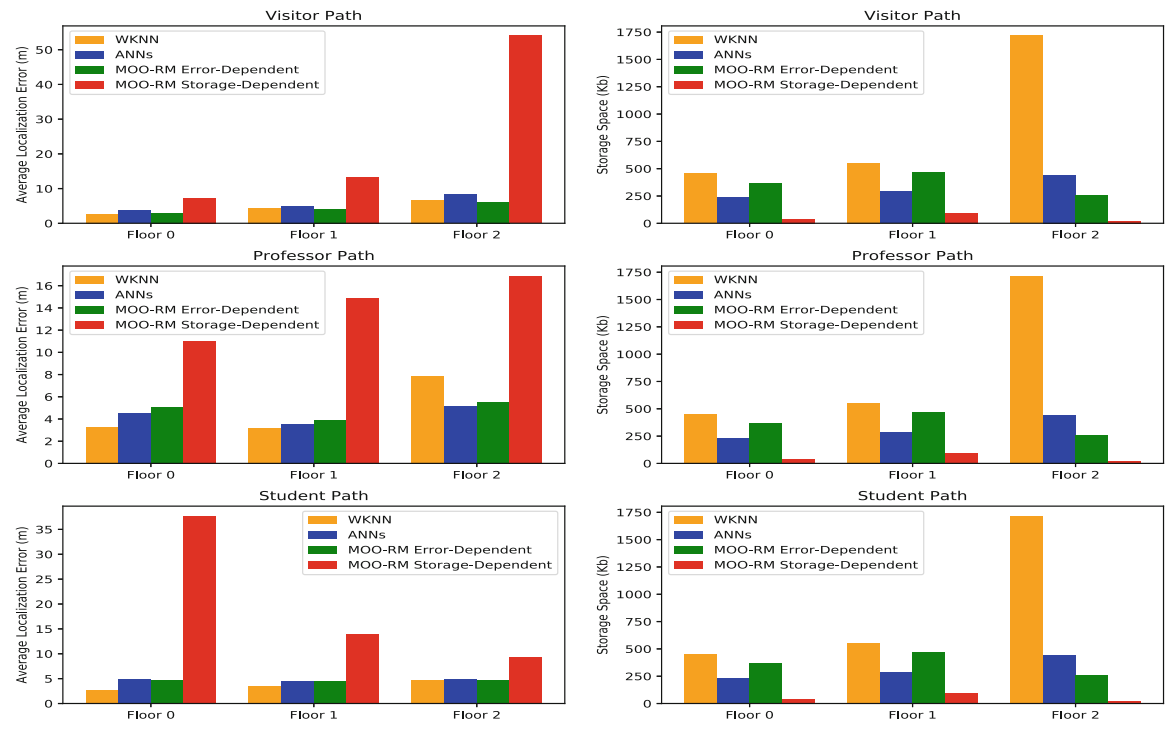

Fig. 5. Paths comparison

\section{Conclusions and Future Work}

In this paper, a novel MOO-RM-based ILS is introduced. MOO-RM provides the user with a variety of solutions in different granularities, each providing different levels of localization error and storage space requirements. This allows the user to localize in an indoor environment based on his preferences e.g. limited storage availability levels might require a smaller model with high localization error trade-off. The experimental results demonstrate the superiority of the proposed approach when compared with conventional client-side and ANN fingerprinting approaches, in terms of resource consumption, while preserving user privacy and maintaining localization accuracy.

Finally, this research study provides solutions based on a fixed ANN structure. As a future challenge, we would like to examine the use of variable structures between the cluster models provided to the MOEA/D. In particular, different ANN model structures result in different levels of storage space requirements and localization error. This will allow us to provide better solutions to smartphone users while navigating based on their preferences.

Acknowledgements. This work is part of the "EnterCY" Integrated Project with project number INTEGRATED/0609/0020, which is co-funded by the European Regional Development Fund and the Cyprus Government through the Research \& Innovation Foundation (RIF) program RESTART 2016-2020. 


\section{References}

1. Gu, Y., Lo, A., Niemegeers, I.: A survey of indoor positioning systems for wireless personal networks. IEEE Commun. Surv. Tutor. 11, 13-32 (2009)

2. Peter, A., Tella, Y., Dams, G.: An overview of indoor localization technologies and applications, June 2015

3. Petrou, L., Larkou, G., Laoudias, C., Zeinalipour-Yazti, D., Panayiotou, C.G.: Crowdsourced indoor localization and navigation with anyplace. In: Proceedings of the 13th International Conference on Information Processing in Sensor Networks, IPSN 2014, Berlin, Germany, 15-17 April, pp. 331-332. IEEE Press (2014)

4. Konstantinidis, A., Chatzimilioudis, G., Zeinalipour-Yazti, D., Mpeis, P., Pelekis, N., Theodoridis, Y.: Privacy-preserving indoor localization on smartphones. IEEE Trans. Knowl. Data Eng. 27(11), 3042-3055 (2015)

5. Zeinalipour-Yazti, D., Laoudias, C.: The anatomy of the anyplace indoor navigation service. SIGSPATIAL Spec. 9, 3-10 (2017)

6. Georgiou, K., Constambeys, T., Laoudias, C., Petrou, L., Chatzimilioudis, G., Zeinalipour-Yazti, D.: Anyplace: a crowdsourced indoor information service. In: 2015 16th IEEE International Conference on Mobile Data Management, vol. 1, pp. 291-294, June 2015

7. Laoudias, C., Constantinou, G., Constantinides, M., Nicolaou, S., ZeinalipourYazti, D., Panayiotou, C.: The airplace indoor positioning platform for android smartphones, July 2012

8. Lymberopoulos, D., et al.: A realistic evaluation and comparison of indoor location technologies: experiences and lessons learned, April 2015

9. Alexandrou, R., Papadopoulos, H., Konstantinidis, A.: Smartphone indoor localization using bio-inspired modeling. In: Yang, X.-S., Zhao, Y.-X. (eds.) NatureInspired Computation in Navigation and Routing Problems. STNC, pp. 149-167. Springer, Singapore (2020). https://doi.org/10.1007/978-981-15-1842-3_7

10. Félix, G., Siller, M., Álvarez, E.N.: A fingerprinting indoor localization algorithm based deep learning. In: 2016 Eighth International Conference on Ubiquitous and Future Networks (ICUFN), pp. 1006-1011, July 2016

11. Wang, X., Gao, L., Mao, S., Pandey, S.: CSI-based fingerprinting for indoor localization: a deep learning approach. IEEE Trans. Veh. Technol. 66, 763-776 (2017)

12. Abbas, M., Elhamshary, M., Rizk, H., Torki, M., Youssef, M.: WiDeep: WiFi-based accurate and robust indoor localization system using deep learning, January 2019

13. Bergenti, F., Monica, S.: A bio-inspired approach to WiFi-based indoor localization. In: Cagnoni, S., Mordonini, M., Pecori, R., Roli, A., Villani, M. (eds.) WIVACE 2018. CCIS, vol. 900, pp. 101-112. Springer, Cham (2019). https://doi. org/10.1007/978-3-030-21733-4_8

14. Ding, G., Tan, Z., Wu, J., Zeng, J., Zhang, L.: Indoor fingerprinting localization and tracking system using particle swarm optimization and Kalman filter. IEICE Trans. Commun. E98.B, 502-514 (2015)

15. Pericleous, S., Konstantinidis, A., Demetriades, A.: A multi-objective indoor localization service for smartphones, April 2019 